\title{
Erratum to: Top local cohomology modules and Gorenstein injectivity with respect to a semidualizing module
}

\author{
Maryam Salimi, Elham Tavasoli, and Siamak Yassemi
}

\begin{abstract}
This note makes a correction to the paper "Top local cohomology modules and Gorenstein injectivity with respect to a semidualizing module".
\end{abstract}

Mathematics Subject Classification (2000). 13D05, 13D45, 18G20.

Keywords. Local cohomology, Semidualizing, Dualizing, $G_{C}$-injective, $C$-injective.

Erratum to: Arch. Math. 98 (2012) 299-305

DOI 10.1007/s00013-012-0371-5

Throughout this paper, $R$ is a commutative Noetherian ring and all modules are unital. In the proof of (iv) $\Rightarrow$ (i) of Theorem 3.1 in the original paper [4], we used explicitly the assumption that $R$ is Cohen-Macaulay. More precisely, the statement (i) in Theorem 3.1 of the original paper implies that $R$ is CohenMacaulay and $\mathrm{H}_{\mathfrak{m}}^{d}(R)$ is a $C$-injective $R$-module. Therefore the corrected version of Theorem 3.1 in the original paper is as follows. (Note that the rest of the paper is unaffected because in all appeals to Theorem 3.1, its new hypothesis that $R$ is Cohen-Macaulay is automatically satisfied.)

Theorem 3.1. (new) Let $(R, \mathfrak{m})$ be a complete local ring of Krull dimension $d$, and let $C$ be a semidualizing $R$-module. Then the following statements are equivalent. 
(i) $C$ is a dualizing $R$-module.

(ii) $R$ is Cohen-Macaulay, and $\mathrm{H}_{\mathfrak{m}}^{d}(R)$ is a $C$-injective $R$-module.

(iii) $R$ is Cohen-Macaulay, and $\mathrm{H}_{\mathfrak{m}}^{d}(R)$ is a $G_{C}$-injective $R$-module.

(iv) $R$ is Cohen-Macaulay, and $\mathrm{H}_{\mathfrak{m}}^{d}(R)$ has finite $G_{C}$-injective dimension.

Proof. (i) $\Rightarrow$ (ii) $\Rightarrow$ (iii) $\Rightarrow$ (iv) follows from the proof of Theorem 3.1.

(iv) $\Rightarrow$ (i) Suppose that $\mathrm{H}_{\mathfrak{m}}^{d}(R)$ has finite $G_{C}$-injective dimension. Since $\mathrm{H}_{\mathfrak{m}}^{d}(R)$ is an Artinian $R$-module, we have that $\mathrm{H}_{\mathfrak{m}}^{d}(R)^{\vee}$ is a finitely generated $R$-module (see [2, Theorem 3.4.7]). By [3, Theorem 2.16], $\operatorname{Gid}_{R \ltimes C}\left(\mathrm{H}_{\mathfrak{m}}^{d}(R)\right)<$ $\infty$. Since $R$ is complete, $\operatorname{Gfd}_{R \ltimes C}\left(\mathrm{H}_{\mathfrak{m}}^{d}(R)^{\vee}\right)<\infty$ by [1, Theorem 4.25]. So $\operatorname{Gpd}_{R \ltimes C}\left(\mathrm{H}_{\mathfrak{m}}^{d}(R)^{\vee}\right)<\infty$, by [1, Theorem 4.23]. In addition, $\mathcal{G} \mathcal{P}_{C^{-}} \mathrm{pd}_{R}$ $\left(\mathrm{H}_{\mathfrak{m}}^{d}(R)^{\vee}\right)=\operatorname{Gpd}_{R \ltimes C}\left(\mathrm{H}_{\mathfrak{m}}^{d}(R)^{\vee}\right)<\infty$, by [3, Theorem 2.16]. The Local Duality Theorem implies that $\mathrm{H}_{\mathfrak{m}}^{d}(R)^{\vee} \cong \Omega$, where $\Omega$ is a canonical module of $R$. By definition, this module is non-zero and finitely generated, so it has finite depth, and it has finite injective dimension because $R$ is Cohen-Macaulay. Since it has finite $G_{C}$-projective dimension, the fact that $C$ is dualizing follows from [5, Corollary 2.9].

Recently, Zargar [6] emphasizes the above mistake.

\section{References}

[1] L. W. Christensen, H. B. Foxby, And H. Holm, Beyond totally reflexive modules and back: a survey on Gorenstein dimensions, "Commutative Algebra Noetherian and non-Noetherian Perspectives", 101-143, Springer, New York, 2011.

[2] E. E. Enochs And O. M. G. Jenda, Relative homological algebra, de Gruyter Expositions in Mathematics, vol. 30, Walter de Gruyter \& Co., Berlin, 2000.

[3] H. Holm and P. Jørgensen, Semidualizing modules and related Gorenstein homological dimension, J. Pure Appl. Algebra, 205 (2006), 423-445.

[4] M. Salimi, E. Tavasoli, And S. Yassemi, Top local cohomology modules and Gorenstein injectivity with respect to a semidualizing module, Arch. Math. (Basel) 98 (2012), 299-305.

[5] S. Sather-Wagstaff and S. Yassemi, Modules of finite homological dimension with respect to a semidualizing module, Arch. Math. (Basel) 93 (2009), 111-121.

[6] M. R. Zargar, Local cohomology modules and Gorenstein injectivity with respect to a semidualizing module, Arch. Math. (Basel), 100 (2013), 25-34.

\section{Maryam SALimi}

Department of Mathematics, Science and Research Branch,

Islamic Azad University,

Tehran, Iran

e-mail: maryamsalimi@ipm.ir 


\section{Elham Tavasoli}

Department of Mathematics, Science and Research Branch, Islamic Azad University,

Tehran, Iran

e-mail: elhamtavasoli@ipm.ir

\section{Siamak Yassemi}

Department of Mathematics,

University of Tehran,

Tehran, Iran

and

School of Mathematics,

Institute for Research in Fundamental Sciences (IPM),

Tehran, Iran

e-mail: yassemi@ipm.ir

Received: 22 October 2012 